\title{
Recognition of Off-line Isolated Handwritten Character Using Counter Propagation Network
}

\author{
W. H. Bangyal, J. Ahmad, and Q. Abbas
}

\begin{abstract}
This paper presents the recognition of off line handwritten English characters using the forward only Counter Propagation network (CPN) algorithm Upper case English alphabets are used in this paper. In the recognition process different people's writing style are gathered. The obtained results in this paper show the effect of the learning rate and recognition accuracy of the $\mathrm{CPN}$ neural network. The algorithm is tested and the recognition rate obtained was over $\mathbf{9 0 \%}$.It shows that proposed algorithm results are reasonable and acceptable for the hand written character recognition application.
\end{abstract}

Index Terms-Handwritten character recognition, counter propagation networks, learning rate, performance

\section{INTRODUCTION}

Artificial Neural Network (ANN) is a computing model of brain, having paralleled distributed processing elements that are learned by adjusting the connected weights between the neurons. Due to its flexibility and strength, it has been now broadly used in different fields such as pattern recognition, decision-making optimization, market analysis, robot intelligence [1].

ANN can be more remarkable as computational processors for different tasks like data compression, classification, combinatorial optimization problem solving," pattern recognition etc [2].

ANN has many advantages over the other classical methods. While having the computational complexity, ANN offered many advantages in pattern recognition adapting a very little context of human intelligence [3].

Pattern recognition has its main objective to classify objects into dissimilar categories and classes. It is a basic part of artificial intelligence and machine vision Pattern recognition is the research area that is used to study and design of the system for the recognition of the different pattern either it is in the form of text, image or numeric [4]

In the last few decades, many applications have been developed to solve the different problems like face recognition and character recognition [5]. From the last few decades, a lot of research is caring on in the field of handwriting recognition [6].

Hand written recognition has become exceptionally popular field in the past decades. Majority of the applications

Manuscript received October 19, 2012; revised January 12, 2013.

W. H.Bangyal is with the Department of Computing and Technology Iqra University Islamabad, Pakitsan (e-mail: waqas_bangyal@hotmail.com).

J. Ahmad is with Abasyn University Peshawar, Pakistan (e-mail: jamil.ahmad@abasyn.edu.pk)

Q. Abbas is with the Department of Computing and Technology Iqra University Islamabad, Pakistan (e-mail: qamar.bhk@gmail.com). fall in to the category of the off-line recognition system that corresponds to already written documents obtained by a scanner or a camera.

Hand written character recognition is becoming a grown-up field at the start of the 21 st century. Off line are now sufficient for particular systems like interpreting handwritten postal addresses on envelopes and reading currency amounts on bank checks [7]

There are two different methodologies used for Character recognition either the on line system or off line system. In On-line character recognition category, the computer recognizes the symbols as they are written on the digitizing tablet. The digitizing tablet acts with one dimension of data and it has resolution of 200 points per inch and a sampling rate of 100 points per second. Where as in the Off-line recognition system, it is performed as the writing process or printing is completed.

In Optical Character Recognition, OCR, it recognize the characters optically than magnetically processed characters Off-line character recognition can be measured as the most common having no special device for writing and signal interpretation is free of signal generation, as in human recognition.

There is lot of research carried out in the field of character recognition for more than three decades [8]. Yet, this character recognition field remains the main demanding dilemma in pattern recognition and all the current existing systems are still restricted to limited applications.

The paper presents the off line handwritten character recognition using the forward only CPN algorithm. For the experimental results twenty six upper case English alphabets are considered in this paper. No constraint is applied on the writing style. This learning rate parameter of the neural network has a great affect on the accuracy of the network results.

In this paper forward only counter propagation network [9] is used., CPN is a classification network in its simplest type, it takes a feature vector as a input vector and gives an output as what it has classified it and the chances of the probability of the classifications being correct. It is combination of unsupervised and supervised learning together proposed by Robert Hecht- Nielson [10], consisting of input layer, competitive layer and output layer. From input layer to competitive layer form the Kohonen network, while from competitive layer to and output layer form the Gross berg network. Using the Winner- Take-All strategy in the competitive learning, one neuron can be winner in the competitive layer and set its status to 1 while other to 0 .

This paper is organized as follows. In Sections II literature survey is given. The Section III gives proposed architecture. 
In Section IV, preprocessing is done. In Section V, experimental results are given and lastly, conclusions are drawn in Section VI.

\section{LITERATURE SURVEY}

This paper presents implementation of the forward only $\mathrm{CPN}$ in the hand written character recognition application. In an effort to determine the applicability of forward only CPN to the problem of hand written character recognition, the effect of the learning rate and recognition accuracy of the CPN neural network is checked.

In [10] the author used CPN Neural Network for on line hand written recognition application and the results of the recognition rates were up to $94 \%$ in the experiments.

In [11] the author have used Neural Network classifier for feature extraction of hand written digits and find the accuracy up to $98 \%$ in the results of the experiments.

In [12] the author have used CPN Neural Network for the on line character recognition classifier and illustrates the feature extraction methodology in detail and the results of the accuracy were above $90 \%$ in the application

In [13] the author have used CPN Neural Network for efficient text recognition system which makes possible the training and recognition of targeted text at the same time. The system can be utilized for any font. It shows $100 \%$ results for computer created images and noise free images

In [14] the author has used BPN Neural Network for un constrained the hand written characters. Results shows the performance of the BPN was experimented more accurate both using momentum term and without using the momentum term.

\section{The Proposed Network ArChitecture}

I have proposed forward only counter propagation network for the recognition of the hand written character recognition application.

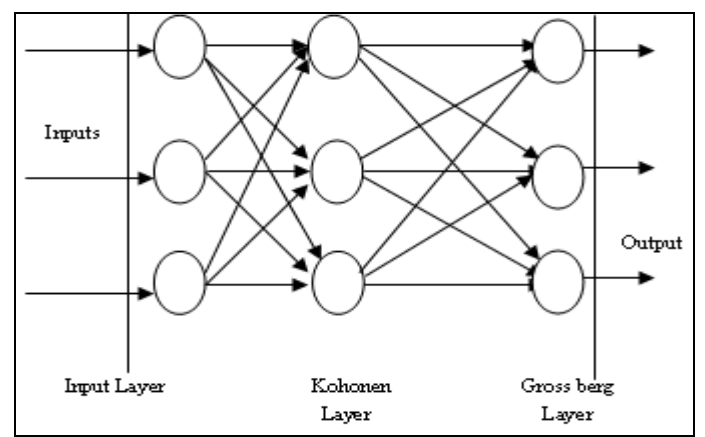

Fig. 1. Hand Written Character Architecture

The forward only CPN network has three layers. First layer is called the input layer. The second layer is known as kohonen (competitive layer) and third layer is called as Gross berg layer.

The overall architecture of the neural network used for hand written character recognition is shown in the Fig. 1.

The characters that are being used here are English alphabets written in the form of a matrix of $10 \times 10$. The parameters can affect the forward only counter propagation network k algorithm in term of the accuracy of result. Several experiments have been performed to look into the effect of using different learning rate. The analysis for the recognition of the hand written character is now done on the basis of different learning rate value that shall be discussed next.

\section{LEARNING / TRAINING PREPROCESSING}

In the paper only twenty six English Upper case alphabets characters have been used. Each candidate was given the instruction to write the characters on writing area. There is no restriction imposed on the content or style of writing. The writers consist of university students, professors, and employees in the university.

In the preprocessing stage consists in selecting the representation space, and the relevant information for the proposed application. In the first stage of preprocessing the gray scale image is transforming in to bi-modal ones (containing only black and white colors).

Generally handwriting recognition system is described by a number of steps including digitization, preprocessing, binarization, training, classification or recognition and testing. Preprocessing techniques focus at reducing the inconsistency that is inborn in cursive and hand printed characters. Some of the preprocessing techniques like scaling, noise elimination, correction that are used to improve the recognition process performance.

The character is captured into digitizing grid into $10 \times 10=100$ digital cells results 100 input vector, each having a single color, black or white. It is important to educate this information into a useful meaningful form to a computer. And the boxes found with character pixels are considered "as on" and the remaining considered as "off" then it will presented to the neural network input for training and recognition purposes.
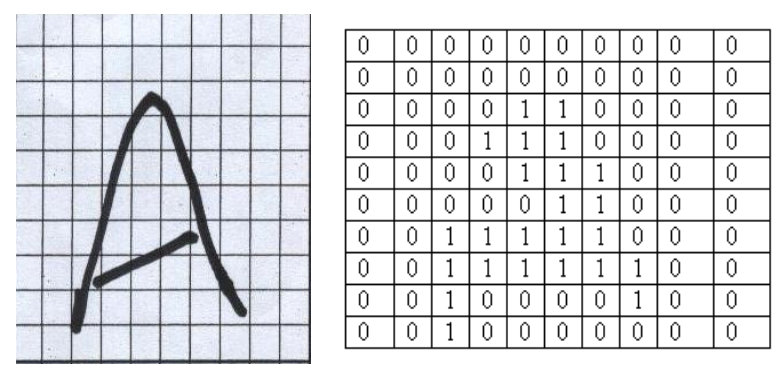

Fig. 2. Binary Representation of Character 'A' and (b) Matrix Representation

The binary matrix is shown in following Fig. 2. The binary 1 show the existence of the character in the cell and binary 0 shows that that there is no character in the cell. A file is maintained of these 1000 hand written upper case English alphabets characters and then used in simulation to get the results. Now this matrix containing binary 0 and 1 and used as input for training and testing of the ANN

For the training I have collected data of about 10000 characters 40 sample of each of the twenty six characters is used to get the results. For effective training of the designed network (to avoid over-fitting), the testing sets of 250 characters are used randomly .A set of examples used only to assess the performance or generalization of a fully-specified classifier [16]. 


\section{EXPERIMENTAL RESULTS}

All testing is performed on the basis of the data samples taken from individuals who did not contributed in the initial process of collecting the training data sets. The accuracy of results for hand written characters shown in the figures

TABLE I: ACCURACY OF THE RESULTS AGAINST DIFFERENT LEARNING RATE

\begin{tabular}{cc}
\multicolumn{2}{c}{ VALUE } \\
\hline Learning Rate & Recognition Rate (\%) \\
\hline 0.9 & 98 \\
\hline 0.8 & 92 \\
\hline 0.7 & 88 \\
\hline 0.6 & 86 \\
\hline 0.5 & 80 \\
\hline
\end{tabular}

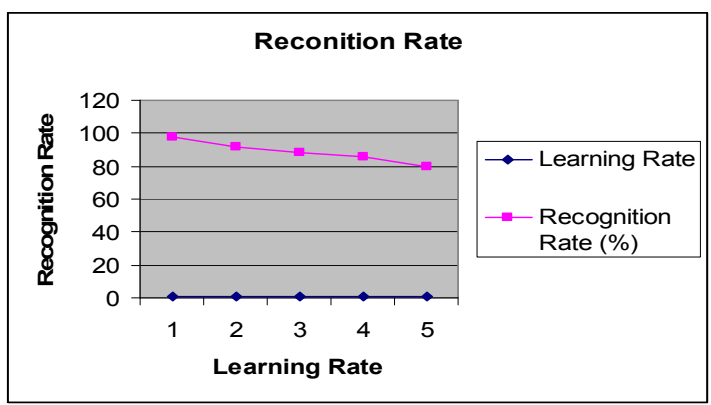

Fig. 3. Simulation result for the accuracy of results against learning rate

From Table I, have the following observations and it shows that learning rate and recognition rate can be analyzed as follows

- The value of accuracy increases as the learning rate increases as shown in corresponding table and graph.

- As in forward only CPN, there is no as such need of training parameters and also it is not an iterative method like BPN which acquired a long time for learning the model. It is also observed that as the training samples/ characters increases, performance of the model also increases. Recognition of the handwritten characters with different learning rate is shown in the Table I. As the learning rate decreases, it decreases the correct recognition, also avoids the system to go for false recognition (FRs). In the recognition process false recognition is an important factor, lesser the false recognition rate, the more reliable the system [17]. Instead of false recognition (FRs), the proposed system goes for recognition failure (RFs) which is not as much of dangerous as false recognition (FRs).

Table II shows the calculation of the processing time of the forward only CPN implementation is carried on an Intel Core $2.13 \mathrm{GHz}$ Processor personal computer running windows XP with the results shown in the figures. It shows the results of the performance of the forward only CPN model for the character recognition application with different number of training samples shown in the table and checked the training time with different learning rate values

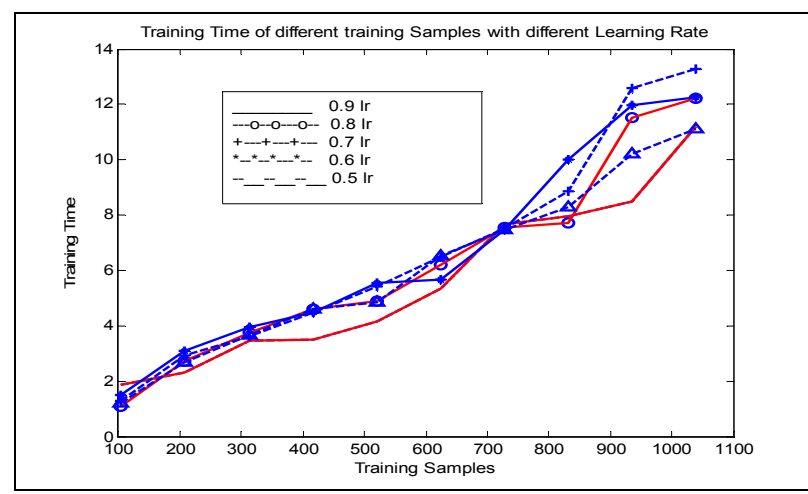

Fig. 4. Simulation result for the accuracy of results against learning rate with different training samples

From Table II, have the following observations and it shows that learning rate, training samples and training time can be analyzed as that value training time increases as the training samples increases as shown in corresponding table and graph. So the training samples affect the training time.

\begin{tabular}{|c|c|c|c|c|c|c|c|c|c|}
\hline \multicolumn{2}{|c|}{ Lr 0.9} & \multicolumn{2}{|c|}{ Lr 0.8} & \multicolumn{2}{|c|}{ Lr 0.7} & \multicolumn{2}{|c|}{ Lr 0.6} & \multicolumn{2}{|c|}{ Lr 0.5} \\
\hline Samples & Time in Secs & Samples & Time in Secs & Samples & Time in Secs & Samples & Time in Secs & Samples & Time in Secs \\
\hline 104 & 1.87 & 104 & 1.1 & 104 & 1.29 & 104 & 1.48 & 104 & 1.21 \\
\hline 208 & 2.3 & 208 & 2.73 & 208 & 2.92 & 208 & 3.1 & 208 & 2.7 \\
\hline 312 & 3.47 & 312 & 3.76 & 312 & 3.63 & 312 & 3.96 & 312 & 3.65 \\
\hline 416 & 3.52 & 416 & 4.62 & 416 & 4.47 & 416 & 4.48 & 416 & 4.6 \\
\hline 520 & 4.15 & 520 & 4.91 & 520 & 5.42 & 520 & 5.54 & 520 & 4.84 \\
\hline 624 & 5.35 & 624 & 6.2 & 624 & 6.5 & 624 & 5.69 & 624 & 6.51 \\
\hline 728 & 7.68 & 728 & 7.54 & 728 & 7.5 & 728 & 7.47 & 728 & 7.49 \\
\hline 832 & 7.98 & 832 & 7.71 & 832 & 8.86 & 832 & 9.99 & 832 & 8.29 \\
\hline 936 & 8.49 & 936 & 11.54 & 936 & 12.6 & 936 & 11.97 & 936 & 10.21 \\
\hline 1040 & 11.19 & 1040 & 12.21 & 1040 & 13.28 & 1040 & 12.26 & 1040 & 11.12 \\
\hline
\end{tabular}

\section{CONCLUSION}

The paper concludes that proposed forward only CPN algorithm has very good capability for the off line hand written character recognition. It is a simple but vigorous algorithm for the classification of the patterns. And from the performance analysis it can be concluded that the learning rate affects the accuracy of the recognition of the hand written character recognition.

\section{REFERENCES}

[1] M. Simner, W. Hulstijn, and P. Girouard (Eds.), "Forensic, developmental and neuropsychological aspects of handwriting," Special issue of of the Journal of Forensic Document Examination, 1994.

[2] R. Plamondon, "Pattern recognition," Special Issue on Automatic Signature Verification, vol. 8, no. 3, June 1994 
[3] G. P. Van Galen and P. Morasso, "Neuromotor control in handwriting and drawing," Acta Psychologica,, vol. 100, no. 1-2, p. 236, 1998.

[4] G. Passalis, I. A. Kakadiaris, T. Theoharis, G. Toderici, and N. Murtuza, "Three-Dimensional face recognition in the Presence of facial expressions: An annotated deformable model approach," IEEE Transactions on Pattern Analysis and Machine Intelligence, vol. 29, no. 4, pp. 640-650, April 2007.

[5] L. Xiaobo, L. Xiaojing, and H. Wei. "Vehicle license plate character recognition," in Proc. IEEE Int. Conf. Neural Networks and Signal Processing, pp. 1066-1069, 2003.

[6] A. R. Khan and D. Muhammad, "A simple segmentation approach for unconstrained cursive handwritten words in conjunction with the neural network," International Journal of Image Processing, vol. 2, issue (3) 29, 2007

[7] H. Nielsen, "Counterpropagation networks," in Proc. IEEE First Int'l Conference on Neural Networks, 1987 II: 19-32.

[8] M. Yusoff, S. A. Rahman, S. Mutalib, and A. Mohamed, "Kohonen neural network performance in license plate number identification," in Proc. International Conference on Electrical Engineering and Informatics Institut Teknologi Bandung, Indonesia, pp. 20-24, 2007.

[9] J. Moreno, G. Sebastián1, M. A. Fernandez, and A. F.Aballero, "A telephone number corrector using acounterpropagation network," in Proc. the Fifth International Conference on Neural Information Processing ICONIP'98, Kitakyushu, Japan, pp. 1-6, 1998.

[10] M. F. Zafar, D. Mohamad, and R. M. Othman, "On-line handwritten character recognition: An implementation of counterpropagation neural net," World Academy of Science, Engineering and Technology, pp. 1-6, 2007.
[11] H. Altun and G. Gelen, "Enhancing performance of MLP/RBF neural classifiers via an multivariate data distribution scheme," Nigde University, Electrical and Electronics Engineering Department Nigde, Turkey, pp. 1-6, 2002.

[12] S. Haykin, Neural Networks, a comprehensive foundation, Second edition, 2005

[13] H. Cecotti and A. Belaid, "Rejection strategy for convolutional neural network by adaptive topology applied to handwritten digits recognition," IEEE, 2005.

[14] E. O. Justice and R. A. Sundayn "An optimum solution for a process control problem (continuous stirred tank reactor) using a hybrid neura network," Journal of Theoretical and Applied Information Technology, vol. 4, no. 10, pp. 1-10, 2008.

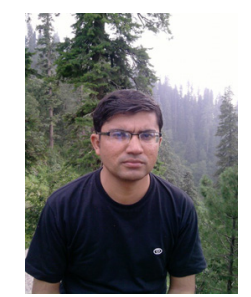

W. H. Bangyal received the BSc degree in mathematics and physics in 2001 from Govt, College Multan, Pakistan, MSc degree in Computer Science in 2003 from Bahauddin Zakariya University Multan, Pakistan. He completed his MS degree in computer science in 2009 from Iqra University Islamabad, Pakitsan. Currently he is a $\mathrm{PhD}$ (computer science) student at the Department of Computing and Technology Iqra University Islamabad, Pakistan. His research interests include pattern recognition, neural networks and evolutionary algorithms. He is an active member of several professional Societies. 